\title{
DISPLAYING QUADRATIC SURFACES ON COMPUTER SCREEN
}

\author{
Ahmed A. Elsonbaty \\ Civil Eng. Dept., Faculty of Engineering, Assiut University, Assiut, Egypt \\ asonbaty2@yahoo.com
}

(Received February 19, 2008 Accepted March 8, 2008)

\begin{abstract}
The problem of picturization of an object is the problem of determination of what lines to draw in a plane in order that the impression conveyed to the eye shall be that of three dimensional object. In this paper computer procedure for generating line drawings of solid objects bounded by quadratic surfaces is described. It embodies an efficient solution to the 'hidden-line' problem, that is, the problem of determining which parts of an opaque object are invisible when the object is viewed from a given view point. In this paper, we present a method to view a quadratic surface of revolution when it is projected using parallel and central projections.
\end{abstract}

KEY WORDS: Projective geometry, central and parallel projection, computer graphics.

\section{INTRODUCTION}

Projection of quadratic surfaces and estimating their visible parts are important in many fields such as computer graphics and photogrammetric application. The surface of some objects may contain quadratic surfaces (such as cylinder cone, sphere, ... etc). And the main aim in photogrammtery, when we have a photo for the object, is to determine the space coordinates of any point appearing in the image plane. To do this, we have to determine at first on which surface the point is located, then find out if it belongs to the visible part of the surface or not. In the following section, we briefly present different types of quadrics

\section{QUADRATIC SURFACES (QUADRICS)}

Quadrics are second-degree algebraic surfaces in three dimensional space $R^{3}$, that is, they are described by polynomials of degree two in the variables $\mathrm{x}, \mathrm{y}$, and $\mathrm{z}$. The general common equation of all quadratics is [1]:

$a_{11} x^{2}+a_{22} y^{2}+a_{33} z^{2}+2 a_{12} x y+2 a_{23} y z+2 a_{13} x z+2 a_{14} x+2 a_{24} y+2 a_{34} z+a_{44}=0$

Where $a_{11}, a_{22}, a_{33}, a_{12}, a_{23}, a_{13}, a_{14}, a_{24}, a_{34}$ and $a_{44}$ are constants, and at least one of the first six does not vanish.

The quadratic surface can be classified into three groups:

1. surfaces without center (such as elliptic paraboloid, hyperbolic paraboloid and parabolic cylinder)

2. surfaces with one center point (such as ellipsoid, one sheeted hyperboloid, twosheeted hyperboloid, cone surface and spheres) 
3. surfaces with infinitely many center points such as hyperbolic and elliptic cylinder.

Obviously, this definition is invariant to the system of coordinates chosen. Indeed, the equation of the surface in any other system of coordinates $x^{\prime} y^{\prime} z^{\prime}$ is obtained from the above equation by substituting $x, y$ and $z$ by linear expressions in $x^{\prime}$, $y^{\prime}$ and $z^{\prime}$. If a quadrics has its center at $\mathrm{O}(0,0,0)$ and its principal axes aligned with $\mathrm{x}^{\prime}$, $\mathrm{y}^{\prime}$ and $\mathrm{z}^{\prime}$, its equation can be simplified into the form (canonical form):

$$
\alpha x^{\prime 2}+\beta y^{\prime 2}+\gamma z^{\prime 2}+\delta=0
$$

Where: $x^{\prime}, y^{\prime}$ and $z^{\prime}$ are linear functions of $x, y$, and $z$.

\section{SURFACES OF REVOLUTION}

If a curve in $\mathrm{R}^{3}$ rotates about a line, it generates a surface of revolution [5]. Let $y=f(x)$ be a curve of the ${ }^{x y}$-plane rotating about the x-axis. Then any point $(x, y)$ of this 0-meridian draws a circle of latitude, parallel to the $z y$-plane, with centre on the $x$-axis and with the radius $|f(x)|$. So the $y$ - and $z$-coordinates of each point on this circle satisfy the equation

$$
y^{2}+z^{2}=[f(x)]^{2}
$$

This equation is thus satisfied by all points $(x, y, z)$ of the surface of revolution and therefore it is the equation of the whole surface of revolution.

More generally, if the equation of the meridian curves in the $x y$-plane is given in the implicit form $F(x, y)=0$, then the equation of the surface of revolution may be written

$$
F\left(x, \sqrt{y^{2}+z^{2}}\right)=0
$$

The most important quadratic surfaces of revolution for our discussion are:

- When the ellipse $\frac{x^{2}}{a^{2}}+\frac{y^{2}}{b^{2}}=1$ rotates about the $\mathrm{x}$-axis, we get the ellipsoid

$$
\frac{x^{2}}{a^{2}}+\frac{y^{2}}{b^{2}}+\frac{z^{2}}{b^{2}}=1
$$

This is a stretched ellipsoid, if $a>b$, and a flattened ellipsoid, if $a<b$, and a sphere of radius a, if. $a=b$

- When the parabola $y^{2}=2 p x \quad$ (with $p$ the latus rectum or the parameter of parabola) rotates about the $x$-axis, we get the paraboloid of revolution

$$
y^{2}+z^{2}=2 p x .
$$

- When we let the conjugate hyperbolas and their common asymptotes 


$$
\frac{x^{2}}{a^{2}}-\frac{y^{2}}{b^{2}}=s
$$

(with $s=1,-1,0)$ rotate about the $x$-axis, we obtain the two-sheeted hyperboloid

$$
\frac{x^{2}}{a^{2}}-\frac{y^{2}}{b^{2}}-\frac{z^{2}}{b^{2}}=1
$$

the one-sheeted hyperboloid

$$
\frac{x^{2}}{a^{2}}-\frac{y^{2}}{b^{2}}-\frac{z^{2}}{b^{2}}=-1
$$

and the cone of revolution

$$
\frac{x^{2}}{a^{2}}-\frac{y^{2}}{b^{2}}-\frac{z^{2}}{b^{2}}=0
$$

which apparently is the common asymptotic cone of both hyperboloids.

\section{NOTATIONS:}

In this paper we will use lower case for scalar variables, lower bold case for vectors and we use the homogeneous coordinate notation $\left(x_{0}: x_{1}: x_{2}: x_{3}\right)$ where the coordinates of any point in Cartesian coordinates are: $\left(x_{1} / x_{0}, x_{2} / x_{0}, x_{3} / x_{0}\right) .\left[x_{O} \neq 0\right]$

\section{DETERMINING THE SILHOUETTE}

In the following, we describe an algorithm to calculate the silhouette of quadrics. The silhouette is the curve on the surface, whose projection embraces the projection of the whole surface. For clarification, we shall explain this method using a hyperboloid surface. This method can also be applied for all quadrics.

Let the homogeneous equation of the hyperboloid be in the form:

$$
a_{1} x_{1}^{2}+a_{2} x_{2}^{2}+a_{3} x_{3}^{2}+a_{0} x_{0}^{2}=0
$$

In case of a hyperboloid of revolution about the $z$-axis, we have

$$
a_{1}=\frac{1}{a^{2}}, a_{2}=\frac{1}{a^{2}}, a_{3}=\frac{-1}{c^{2}}, \text { and } a_{0}=-1
$$

To compute visibility for the surface, we determine the polar plane w.r.t. the center of projection $S\left(\bar{x}_{O}: \bar{x}_{1}: \bar{x}_{2}: \bar{x}_{3}\right)$ (see figure 1), then find its curve of intersection with the surface. This curve called silhouette.

The silhouette clearly separates the visible and the invisible parts. The equation of polar plane $\mathcal{E}$ has the form:

$$
a_{1} x_{1} \bar{x}_{1}+a_{2} x_{2} \bar{x}_{2}+a_{3} x_{3} \bar{x}_{3}+a_{0} x_{0} \bar{x}_{0}=0
$$

Note: in case of parallel projection $\bar{x}_{0}=0$, but in case of central projection the coordinates of center of projection $\mathrm{S}$ can be: $\bar{x}_{0}=1, \bar{x}_{1}=x, \bar{x}_{2}=y, \bar{x}_{3}=z$. 


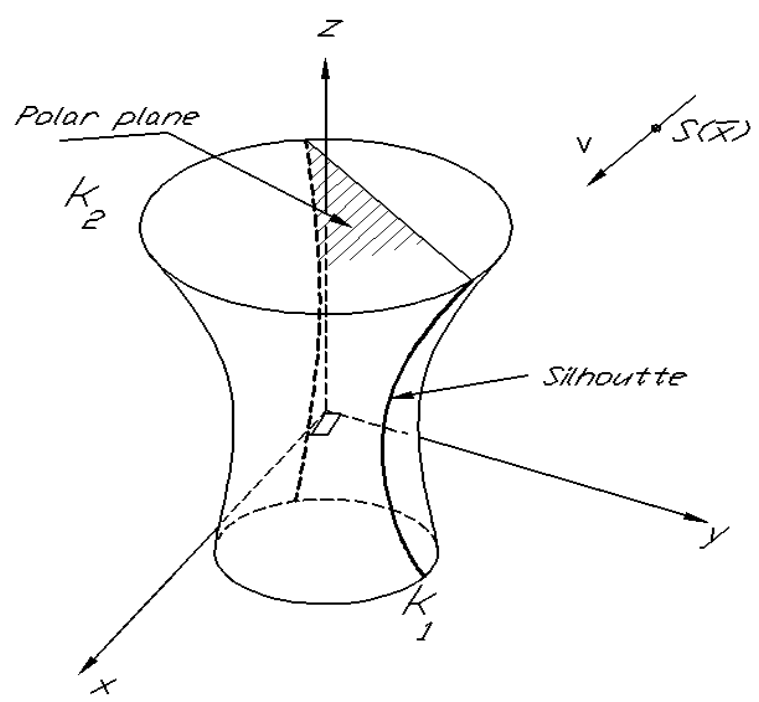

Figure (1): Silhouette of the surface

From equation (12) we get:

$$
x_{1}=-\frac{a_{2} x_{2} \bar{x}_{2}+a_{3} x_{3} \bar{x}_{3}+a_{0} x_{0} \bar{x}_{0}}{a_{1} \bar{x}_{1}}
$$

Substituting $x_{1}$ in equation (11) we get:

$$
d_{1} x_{2}^{2}+d_{2} x_{3}^{2}+x_{2} x_{3} d_{3}+d_{4} x_{2}+d_{5} x_{3}+d_{6}=0
$$

Where $d_{1}, d_{2}, d_{3}, d_{4}, d_{5}$ and $d_{6}$ are constants.

Equation (14) represents a quadratic cylinder through the silhouette with generators parallel to the $x$-axis

Note: In case $\bar{x}_{1}=0$, a similar procedure can be done by substituting for $\bar{x}_{2}$ or $\bar{x}_{3}$ instead of $\bar{x}_{1}$. The origin is excluded.

Since for the silhouette $z_{1} \leq x_{3} \leq z_{2} \quad\left(z=z_{1}\right.$ and $z=z_{2}$ are planes of upper and lower bases of hyperboloid), we can assume a value for $x_{3}$ and substitute it in equation (14) to get a quadratic equation in $x_{2}$ having the form:

$a_{-} x_{2}^{2}+b_{-} x_{2}+c_{-}=0$

Then,

$$
x_{2}=\frac{-b \pm \sqrt{b_{-}^{2}-4 a_{-} c_{-}}}{2 a_{-}}
$$

Substituting $x_{2}$ in (13), two values of $x_{1}$ can be obtained. The point $p$ whose coordinates are $\left(x_{1}, x_{2}, x_{3}\right)$ is located on the silhouette curve. As $x_{3}$ varies from $z_{1}$ to $z_{2}$, the point $p$ traces out the silhouette curve.

In the special case, when the view center $S(0,0, z)$ is located on $z$-axis, then the silhouette is a circle (intersection of the polar plane $\mathcal{E}$ with surface) with radius 


$$
R_{-}=a \sqrt{1+\frac{\bar{x}_{0}^{2}}{\bar{x}_{3}^{2}}}
$$

In case of parallel projection $\bar{x}_{0}=0$ then $R_{-}=a$

Note: The silhouette is in general a conic section. In case it is not extended to infinity, its upper or lower limits may lie between $z=z_{1}$ and $z=z_{2}$. Here we have to determine these points by using equation (15). The quantity under the radical root is zero for this case, from which $z$ can be calculated (see Fig. 2).

\section{CIRCULAR BASES}

The two bases of hyperboloid are circles with radii $R_{1}$ and $R_{2}$ located in the planes $z=z_{1}$ and $z=z_{2}$ respectively, where:

$$
R_{1}=a \sqrt{1+\frac{z_{1}^{2}}{c^{2}}} \text { and } R_{2}=a \sqrt{1+\frac{z_{2}^{2}}{c^{2}}}
$$

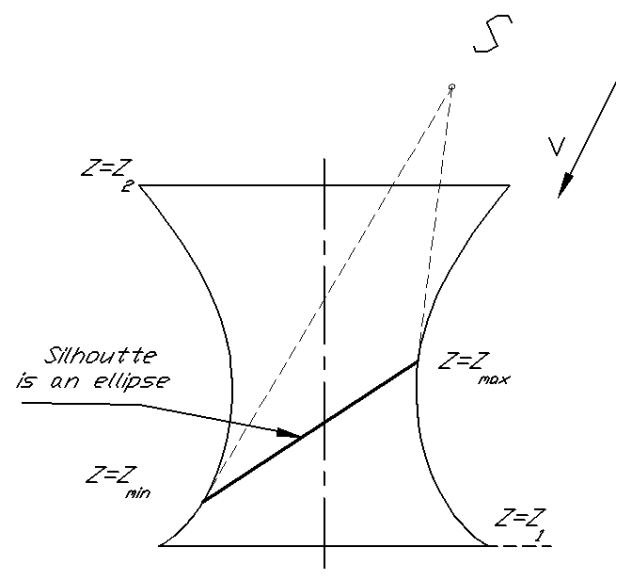

Figure (2): $S$ inside the surface
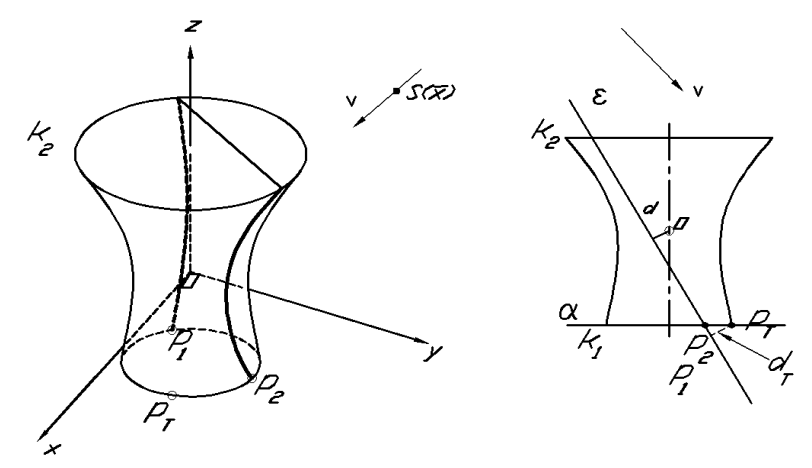

Figure (3): Intersection of polar plane with surface 


\section{VISIBILITY OF THE SURFACES}

In general, the visible part of the projection's outline surface consists of the projection of three parts: silhouette, one of the circular bases and part of the other circular base.

\subsection{In case of parallel projection}

\section{A) Silhouette Curve}

Silhouette curve is generally visible. In parallel projection the polar-plane passes through the center of the surface (origin), since $S\left(0: \bar{x}_{1}: \bar{x}_{2}: \bar{x}_{3}\right)$. .

The equation of polar plane $\varepsilon$ can be written in the vector form as:

$$
\begin{aligned}
& \varepsilon: \boldsymbol{n} \cdot \boldsymbol{x}=d \quad a_{1} x_{1} \bar{x}_{1}+a_{2} x_{2} \bar{x}_{2}+a_{3} x_{3} \bar{x}_{3}=0 . \\
& d=\frac{-a_{0} x_{0} \bar{x}_{0}}{\sqrt{\left(a_{1} \bar{x}_{1}\right)^{2}+\left(a_{2} \bar{x}_{2}\right)^{2}+\left(a_{3} \bar{x}_{3}\right)^{2}}}=0
\end{aligned}
$$

\section{B) Circular bases}

There are two circles; one of them and a part of the other circle are visible. At first, we determine which one is visible. This depends on the direction of view vector $\boldsymbol{v}$.

When $v \cdot z<0$, the upper circle $k_{2}$ is visible and the other one $k_{1}$ is invisible. The points $P_{1}$ and $P_{2}$ of intersection of polar plane and circle $k_{1}$ are separated visible from the invisible parts of $k_{1}$.
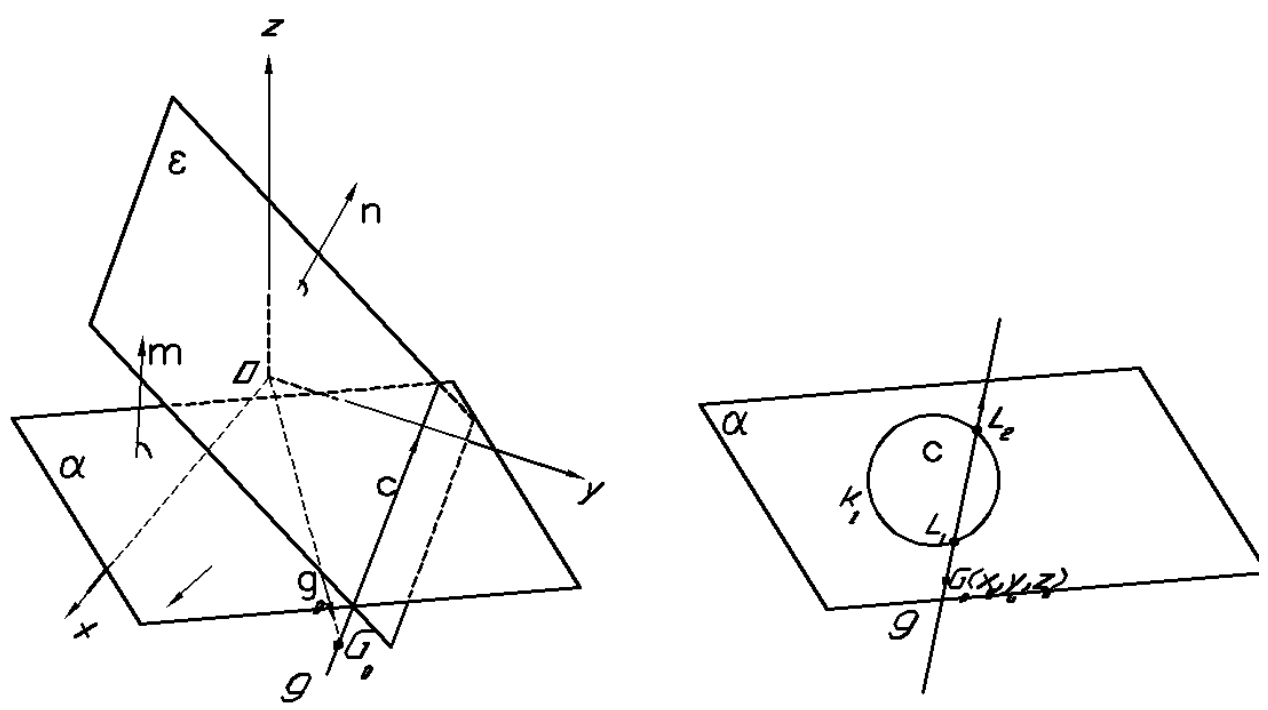

Figure (4): line of intersection between $\mathcal{E}$ and $\alpha$ 
The following procedure illustrates how these points can be calculated.

[1] determine the line of intersection $g$ between polar- plane $\varepsilon$ and the plane $\alpha$ containing circle $k_{1}$

two unbounded non-parallel planes such as $\varepsilon: \boldsymbol{n} \cdot \boldsymbol{x}=d$ and $\alpha: \boldsymbol{m} \cdot \boldsymbol{x}=d_{1}$ intersect in a straight line $g$ in the form:

$$
g: \boldsymbol{x}=\boldsymbol{g}_{0}+\lambda \boldsymbol{c}
$$

$g$ must be orthogonal to both vectors $\boldsymbol{n}, \boldsymbol{m}$, the direction $\boldsymbol{c}$ of $g$ can be calculated as

$$
\boldsymbol{c}=\boldsymbol{n} \times \boldsymbol{m}
$$

$g \subset \alpha, \mathcal{E}$ implies that $\boldsymbol{n} \cdot \boldsymbol{c}=\boldsymbol{m} \cdot \boldsymbol{c}=\boldsymbol{0}$. Now let:

$$
c^{*}=\boldsymbol{x} \times \boldsymbol{c}
$$

From the theorems of vector algebra it is known that [3]

$$
\boldsymbol{c}^{*}=d_{1} \boldsymbol{n}-d \boldsymbol{m} \text {. }
$$

and the position vector for the pedal point $G_{0}$ on $g$ with respect to the origin reads

$$
\boldsymbol{g}_{0}=\frac{\boldsymbol{c} \times \boldsymbol{c}^{*}}{\boldsymbol{c} \cdot \boldsymbol{c}}
$$

[2] Intersection points between $g$ and circle $k_{1}$

The equation of the circle, located in plane $\alpha: z=z_{1}$ can be written as

$$
x^{2}+y^{2}=\left(1+\frac{z_{1}^{2}}{c^{2}}\right) a^{2}
$$

Let $L\left(x_{L}, y_{L}, z_{1}\right)$ be one of the points of intersection between $g$ and $k_{1}$. Since point $L$ lies on line $g$, its coordinates satisfy equation of $g(20)$. Then the base vector $l$ of this point is

$$
\boldsymbol{l}=\boldsymbol{g}_{\boldsymbol{0}}+\lambda \boldsymbol{c}
$$

Also this point $L$ lies on circle $k_{1}$, its coordinates satisfy the equation (22).

$$
\left(x_{G}+\lambda c_{1}\right)^{2}+\left(y_{G}+\lambda c_{2}\right)^{2}=\left(1+\frac{z_{1}^{2}}{c^{2}}\right) a^{2}=R^{2}
$$

Where $x_{G}, y_{G}$ are coordinates of $G_{O}\left(x_{G}, y_{G}\right)$ and $c_{1}, c_{2}$ are components of $\mathbf{c} ; \lambda$ is the only unknown in the above equation, so we can put it in the form:

$$
a_{-} \lambda^{2}+b_{-} \lambda+c_{-}=0
$$

Where $a_{-}, b_{-}$and $c_{-}$are constants. Since the above equation is quadratic equation in $\lambda$, there are two solutions for $\lambda\left(\lambda_{1}, \lambda_{2}\right)$. By substituting about the values of $\lambda$, the points of intersection $L_{1}, L_{2}$ can be obtained. 


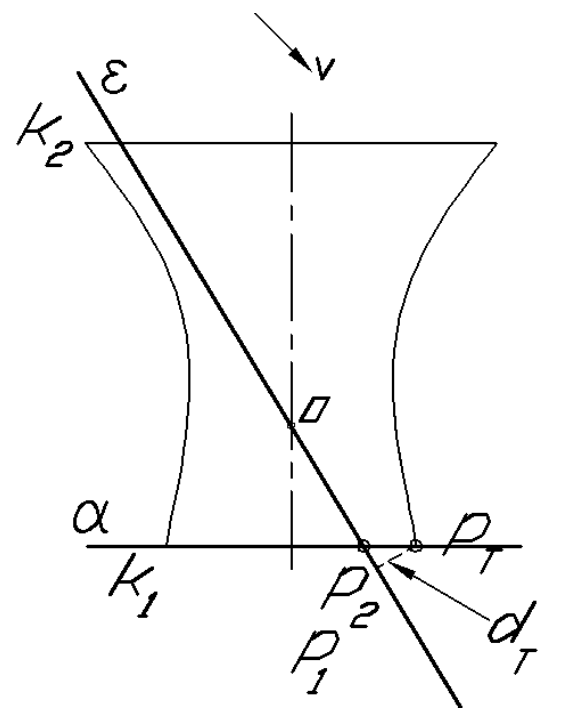

Figure (5): Relation between $P_{T}$ and $\varepsilon$

The points $L_{1}, L_{2}$ divide the circle $k_{1}$ into two parts. One of them is visible and other is not. To determine which part is visible, we use any point located on any part of them, called test point $P_{T}$. The oriented distance $d_{T}$ between point $P_{T}$ (with base vector $\boldsymbol{p}_{T}$ ) and plane $\mathcal{E}$ is

$d_{T}=\boldsymbol{p}_{\boldsymbol{T}} \cdot \boldsymbol{n}-d$

When $d_{T}>0$, then the part passes through $P_{T}$ is visible

\section{SPECIAL CASES}

In some cases the line $g$ does not intersect circle $k_{1}$ as shown in Fig. 6. There are two possible cases:

1. The projection of the two circles $k_{1}^{p}, k_{2}^{p}$ will intersect. The points of intersection can be calculated as follows:

Assume $T^{p}$ is one of intersection points between $k_{1}^{p}$ and $k_{2}^{p}$. this means the point $T^{p}$ is projection of two points one $(T)$ located on upper circle $k_{2}$ and other one $T^{\prime}$ located on the lower circle $k_{1}$, and the line passes them is parallel to view vector $\boldsymbol{v}$.(see Fig. 7)

Since $T\left(x, y, z_{2}\right)$ and $T^{\prime}\left(x^{\prime}, y^{\prime}, z_{1}\right)$ are located on $k_{2}$ and $k_{1}$ respectively, then

$$
\begin{aligned}
& x^{2}+y^{2}=R_{2}^{2}, \\
& x^{\prime 2}+y^{\prime 2}=R_{1}^{2}
\end{aligned}
$$




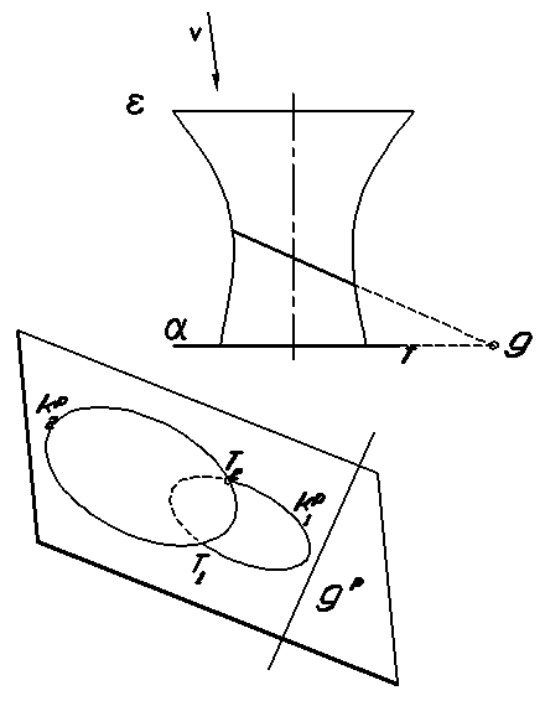

(a)

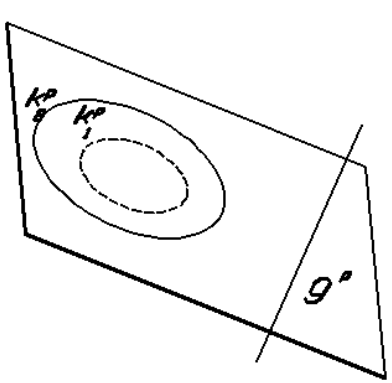

(b)

Figure (6): special cases of Projection of circular bases
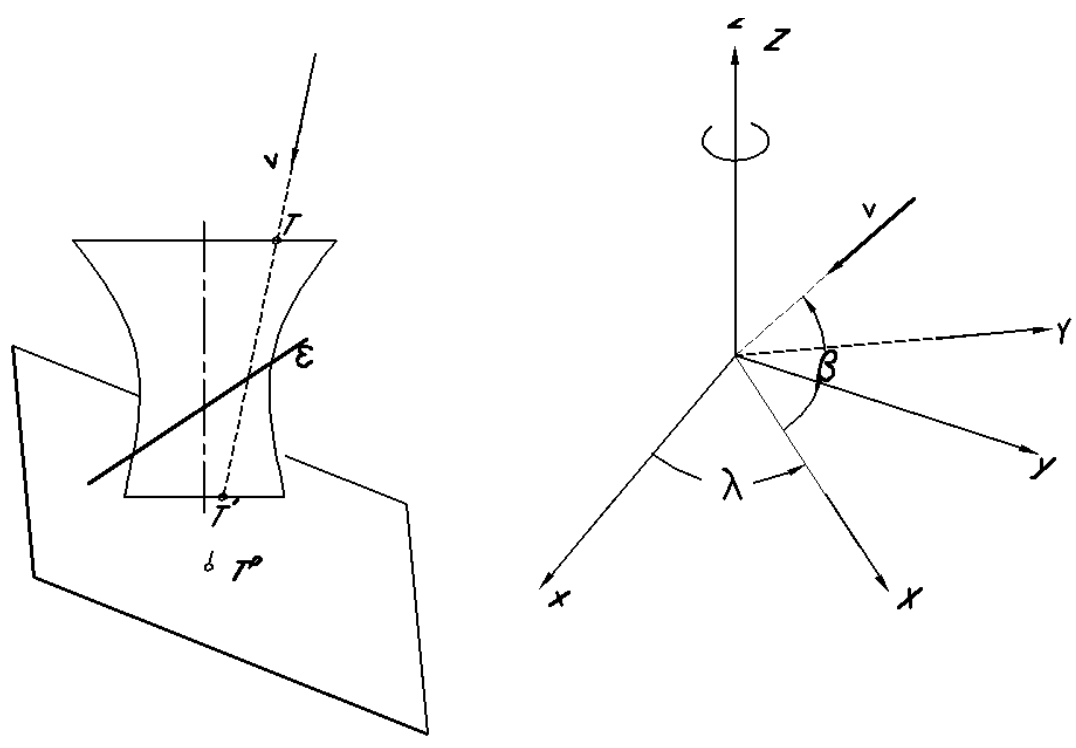

Figure (7): Points of intersection of the projection of the circular bases

Let the direction of view be defined by the two angles $(\lambda$ and $\beta$ ) relative to $x y z$ system [4]. Where angle $\lambda$ is the angle between $x$-axis and the projection of view vector on $x y$-plane and angle $\beta$ is the angle between $x y$-plane and view vector. (see figure7). The vector components of $\boldsymbol{v}$ are:

$$
\boldsymbol{v}(-\cos \lambda \cos \beta,-\sin \lambda \cos \beta,-\sin \beta)
$$


Since the direction of the line passing through $T$ and $T^{\prime}$ is parallel to view vector $\boldsymbol{v}$ then

$$
\begin{aligned}
& \frac{x^{\prime}-x}{l}=-\cos \lambda \cos \beta, \\
& \frac{y^{\prime}-y}{l}=-\sin \lambda \cos \beta, \\
& \frac{z^{\prime}-z}{l}=-\sin \beta .
\end{aligned}
$$

Where $l=\sqrt{\left(x^{\prime}-x\right)^{2}+\left(y^{\prime}-y\right)^{2}\left(z^{\prime}-z\right)^{2}}$

For simplicity, suppose that the rectangular $x y z$ - coordinate system is rotated around its $z$-axis counterclockwise through an angle $\lambda$ (figure 7), we obtain the new coordinate system $X, Y, Z$. The new coordinates $X, Y, Z$ of any point can be computed from old coordinates $x y z$ by.

$$
\left[\begin{array}{l}
X \\
Y \\
Z
\end{array}\right]=\left[\begin{array}{ccc}
\cos \lambda & \sin \lambda & 0 \\
-\sin \lambda & \cos \lambda & 0 \\
0 & 0 & 1
\end{array}\right]\left[\begin{array}{l}
x \\
y \\
z
\end{array}\right]
$$

The inverse transformation is:

$$
\left[\begin{array}{l}
x \\
y \\
z
\end{array}\right]=\left[\begin{array}{ccc}
\cos \lambda & -\sin \lambda & 0 \\
\sin \lambda & \cos \lambda & 0 \\
0 & 0 & 1
\end{array}\right]\left[\begin{array}{l}
X \\
Y \\
Z
\end{array}\right]
$$

The equation (25) can be rewritten as

$$
\begin{aligned}
& \frac{X^{\prime}-X}{l}=-\cos \beta, \\
& \frac{Y^{\prime}-Y}{l}=0, \\
& \frac{Z^{\prime}-Z}{l}=-\sin \beta .
\end{aligned}
$$

By solving equation (24)and (27), we can get, after some reductions, the coordinates of $(T)$ and $T^{\prime}$ relative to $X, Y, Z$-system.

$$
\begin{aligned}
& X^{\prime}=X_{1}^{\prime}=X^{\prime}{ }_{2}=\frac{R_{2}^{2}-R_{1}^{2}}{2 c_{-}}-\frac{c_{-}}{2}, \\
& Y^{\prime}{ }_{1}=\sqrt{R_{1}^{2}-X^{\prime 2}} \text { and } \\
& Y^{\prime}{ }_{2}=-\sqrt{R_{1}^{2}-X^{\prime 2}}
\end{aligned}
$$


Where $c_{-}=\frac{\cos ^{2} \beta}{1-\cos ^{2} \beta}\left(z_{1}-z_{2}\right)^{2}=$ const

The coordinate of $T\left(x, y, z_{2}\right)$ and $T^{\prime}\left(x^{\prime}, y^{\prime}, z_{1}\right)$ can be obtained from equation (27)

2. The projection of the two circles $k_{1}^{p}, k_{2}^{p}$ will not intersect.

When $R_{1}^{2}-\bar{x}^{\prime 2}<0$, the projection of the two circles $k_{1}$ and $k_{2}$ will not intersect.

In this case, we calculate $\boldsymbol{v} \cdot \boldsymbol{z}$, then

$\circ \quad$ if $\boldsymbol{v} \cdot \boldsymbol{z}<0$, then

- $\quad k_{2}^{p}$ (the projection of circle $k_{2}$ ) is visible.

- $\quad$ For the other circle $k_{1}^{p}$, we compare the radius of $k_{1}$ with that of $k_{2}$

- $\quad$ if $R_{1}<R_{2}$, then $k_{1}^{p}$ is invisible else it is visible (the projection of silhouette is imaginary) (see figure 8 )
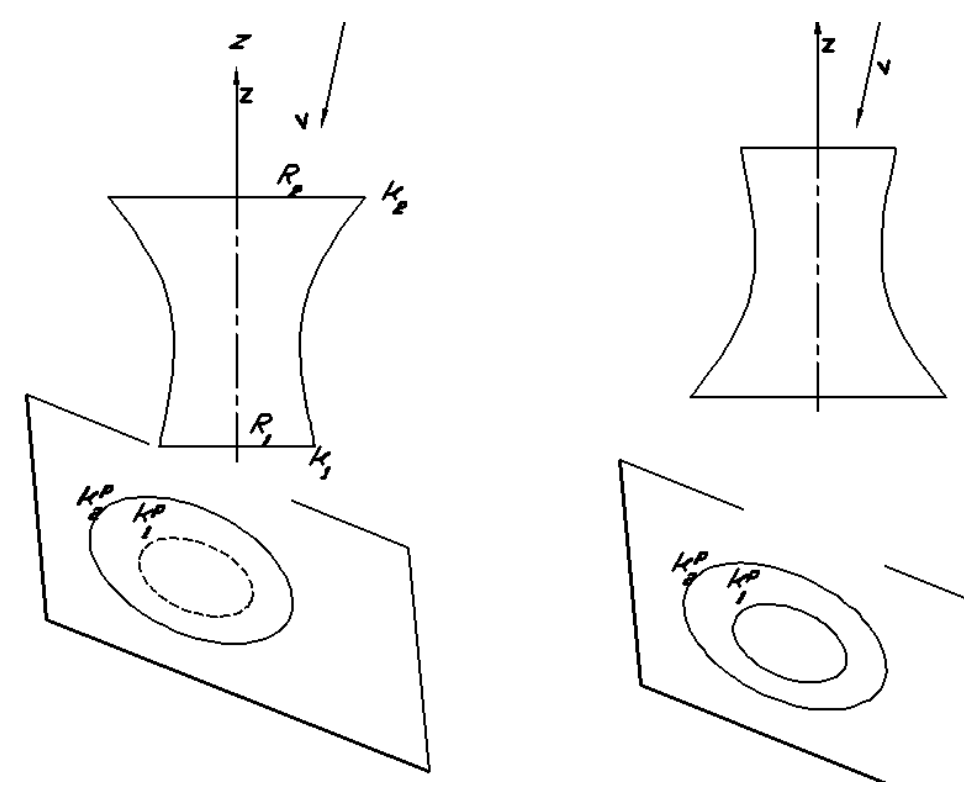

Figure (8): Relative position between the two circular bases

\subsection{In case of central projection}

Let $S$ be the center of projection and its coordinates relative to $x y z$-system be $\left(\left(x_{S}, y_{S}, z_{S}\right)\right.$ and in homogeneous form be $\left(\bar{x}_{0}: \bar{x}_{1}: \bar{x}_{2}: x_{3}\right)$, where $\bar{x}_{0}=1$.

The equation of polar - plane will be in the form:

$$
\varepsilon: \frac{\bar{x}_{1}}{a^{2}} x_{1}+\frac{\bar{x}_{2}}{a^{2}} x_{2}-\frac{\bar{x}_{3}}{c^{2}} x_{3}=\bar{x}_{0} x_{0}=1
$$

The above equation can be rewritten in the form:

$$
\varepsilon: a_{-} x_{1}+b_{-} x_{2}+c_{-} x_{3}=1
$$


The oriented distance

$$
d=\frac{1}{\sqrt{a_{-}{ }^{2}+b_{-}{ }^{2}+c_{-}{ }^{2}}}
$$

from the origin and plane $\mathcal{E}$ is always positive. This means that $\mathcal{E}$ is located between origin $O$ and center point of perspective $S$.

In central projection, we consider that the polar plane is the projection plane. So, the points located between the center $\mathrm{S}$ and polar-plane are visible, in other words, when the oriented distance between point $P$ (with a base vector $\boldsymbol{p}$ ) and polar plane $\mathcal{E}$ is positive $(\overline{P \varepsilon}>0$ or $\quad \boldsymbol{p} \cdot \boldsymbol{n}-\boldsymbol{d}>0$.)
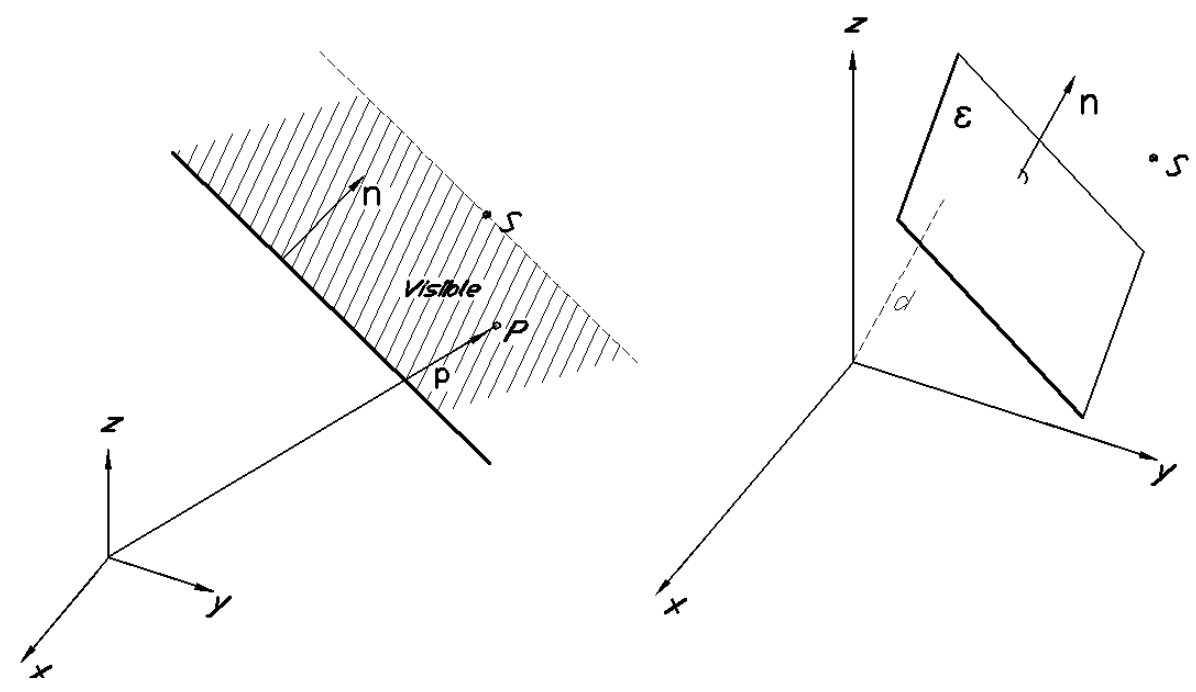

Figure (9): Central Projection

\section{CONCLUSIONS}

Projection of quadratic surfaces and estimating their visible parts are important in many fields such as computer graphics and photogrammetric applications.

In this work, a new technique is described to display quadratic surfaces such as hyperboloid, sphere...etc under parallel and central projection. At first, the polar plane is determined, then its curve of intersection with the surface is found. This curve is called silhouette. The silhouette clearly separates visible and invisible parts and is in general a conic section.

I hope that this method will be useful for researchers who are interested in work on visibility.

\section{REFERENCES}

[1]G.Farin, Curve and Surface for computer Aided Geometric Design, Academic Press, New York 1990. 
[2] A. Elsonbaty, H. Stachel, Generating Solids by Sweeping Polyhedra, J.Geometry and Graphics 1 (1997), 13-21

[3] H. Anton, Elementary Linear Algebra, Ninth Edition, John Wiley \&bSons, Inc (2005).

[4] H. Stachel, Methoden des CAD, Vorlesungsskriptum 1995.

[5] H. Stachel, Darstellende Geometrie, Vorlesungsskriptum 1994

\section{تمثيل مجسمات الارجة الثانية على الكمبيوتر}

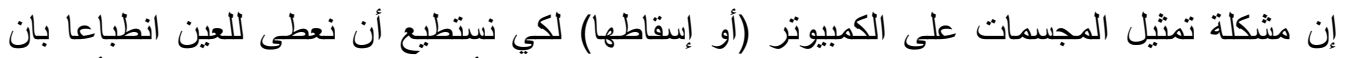

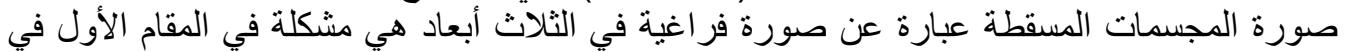

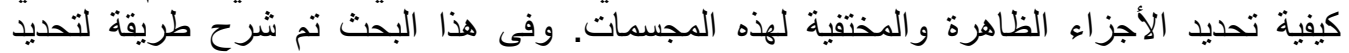
الأجز اء الظاهرة و المختفية لمجسمات الدرجة الثانية وذلك في حالة إسقاطها إسقاطا متوازيا أو مركزيا. 